\title{
Lateral Incision Surgery for Pilonidal Sinus: Death of a Dogma
}

\author{
Mark Cheetham
}

Published online: 16 December 2011

(C) Société Internationale de Chirurgie 2011

\section{Introduction}

Pilonidal sinus disease is common, but there is no universally accepted surgical treatment for the disease. Over the ages, operative solutions have varied from minimal treatment, such as proposed by Lord and Millar [1] and Bascom [2], to the "shock and awe" tactics of wide excision with flap reconstruction [3]. It could be argued that in some cases the results of surgery were worse than the disease.

During the last decade there has been an emerging consensus that operations with a lateral incision are more effective than excision and closure in the midline. The evidence for this comes from several randomized controlled trials and a subsequent meta-analysis [4]. The theory is that shearing forces exerted on the skin by walking are at their greatest within the natal cleft and that there is less opening force exerted on wounds placed lateral to this point. Thus, operations such as the Karydakis procedure [5], Bascom's procedures [2, 6], and rhomboid rotational flaps [7] seem to have a better rate of healing and lower recurrence rates than simple closure in the midline.

In the study published in this issue, Okuş et al. [8] compared patients having the Limberg flap procedure with an operation they call "tension-free primary closure." Perhaps surprisingly, the rate of recurrent disease was similar in the two groups $(4.1 \%$ for the Limberg flap group and $4.3 \%$ for the tension-free primary closure group). This result challenges the notion that a lateral incision is associated with better outcomes following pilonidal sinus surgery.

\section{Cheetham ( $\square)$}

Department of Colorectal Surgery, Royal Shrewsbury Hospital, Mytton Oak Road, Shrewsbury SY3 8XQ, UK

e-mail: mark.cheetham@sath.nhs.uk
Rather than the site of the incision (lateral versus midline) being the primary factor for success, it is equally possible that wide mobilization of skin flaps performed during a Limberg flap or Karydakis procedure results in tension-free closure and therefore an improved outcome. If this is the case, the tension-free primary closure operation described here by Okuş and colleagues merits further investigation. The procedure seems simple and easy to perform. Additionally, the cosmetic results appear to be excellent. Perhaps it should be the procedure of choice for most primary pilonidal disease, reserving more complex operations for recurrent or advanced disease. Time will tell.

\section{References}

1. Lord PH, Millar DM (1965) Pilonidal sinus: a simple treatment. Br J Surg 52:298-300

2. Bascom J (1983) Pilonidal disease: long-term results of follicle removal. Dis Colon Rectum 26:800-807

3. Kapan M, Kapan S, Pekmezci S et al (2002) Sacrococcygeal pilonidal sinus disease with Limberg flap repair. Tech Coloproctol 6:27-32

4. McCallum I, King PM, Bruce J (2007) Healing by primary versus secondary intention after surgical treatment for pilonidal sinus. Cochrane Database Syst Rev (4):CD006213

5. Karydakis GE (1973) New approach to the problem of pilonidal sinus. Lancet 2:1414-1415

6. Bascom J, Bascom T (2002) Failed pilonidal surgery: new paradigm and new operation leading to cures. Arch Surg 137:1146-1150 discussion 1151

7. Daphan C, Tekelioglu MH, Sayilgan C (2004) Limberg flap repair for pilonidal sinus disease. Dis Colon Rectum 47:233-237

8. Okuş A, Sevinç B, Karahan Ö, et al (2011) Comparison of Limberg flap and tension-free primary closure during pilonidal sinus surgery. World J Surg. doi:10.1007/s00268-011-1333-y 